\title{
On the Collaboration Uncapacitated Arc Routing Problem
}

\author{
Elena Fernández ${ }^{* 1}$, Dario Fontana ${ }^{\dagger 2}$, and M. Grazia Speranza ${ }^{\ddagger 2}$ \\ ${ }^{1}$ Department of Statistics and Operation Research, Universitat Politècnica de Catalunya-BcnTech, Spain \\ ${ }^{2}$ Department of Economics and Management, University of Brescia, Italy
}

\begin{abstract}
This paper introduces a new arc routing problem for the optimization of a collaboration scheme among carriers. This yields to the study of a profitable uncapacitated arc routing problem with multiple depots, where carriers collaborate to improve the profit gained. In the first model the goal is the maximization of the total profit of the coalition of carriers, independently of the individual profit of each carrier. Then, a lower bound on the individual profit of each carrier is included. This lower bound may represent the profit of the carrier in the case no collaboration is implemented. The models are formulated as integer linear programs and solved through a branch-and-cut algorithm. Theoretical results, concerning the computational complexity, the impact of collaboration on profit and a game theoretical perspective, are provided. The models are tested on a set of 971 instances generated from 118 benchmark instances for the Privatized Rural Postman problem, with up to 102 vertices. All the 971 instances are solved to optimality within few seconds.
\end{abstract}

\section{Introduction}

Collaboration among carriers becomes more and more valuable because of surging pressures to improve profitability and to reduce costs. Nowadays, collaborative transportation is regarded as one of the major trends in transportation research. Indeed, increasing carrier insurance and fuel costs combined with a more intense market competition lead carriers to look for new and more efficient solutions. Primarily, carriers

\footnotetext{
*e.fernandez@upc.edu

†dario.fontana@unibs.it

¥speranza@eco.unibs.it
} 
focus on reducing costs looking for efficient route planning and scheduling. These costs are strongly correlated with the location of customers. Whereas a carrier would benefit from having its customers concentrated in the same area, for a number of reasons they may end up being geographically dispersed. This forces the carrier to create long routes for its vehicles, with associated high cost in terms of vehicles usage and drivers time. It is often the case that customers that are inconveniently located for a carrier are conveniently located for a different carrier. Thus, a collaborating set of carriers can redistribute the customers, opening up, through collaboration, cost saving opportunities otherwise non achievable.

In general, there are different types of carriers: general, regional or functional. The general carrier is non specialized and has the assets and the logistics to serve all its customers taking care of all kinds of item distributions. Instead, a regional carrier is more bound to a defined geographical service area whereas a functional carrier serves a specific market or specific goods that require a specialization in transportation. Hence, for instance, a regional carrier can rely on a general one to serve customers outside its service area, or a general carrier can choose to handle particular goods (such as furniture, frozen foods) through a functional carrier.

Logistic collaboration can be pushed further considering that it allows carriers to increase the average load of the vehicles. In fact, also in the case the customers are located in the same area, the load to be delivered in a trip by a carrier may be substantially lower than the vehicle capacity and make the individual trip non profitable. A carrier that has to deliver a certain amount of goods that fills only part of the capacity of its smallest vehicle may borrow a vehicle of the right size from another carrier or transfer the load on a vehicle of another carrier traveling to the same area at the same time.

Increasing attention to the environmental impact of emissions in cities represents an additional strong motivation to study collaboration among carriers, since local authorities increasingly push carriers to find new policies and new technological and logistical solutions that improve city logistics. In [31] challenges and pressures faced by carriers to cooperate to make urban freight transport more efficient are pointed out, and best practices actually brought into practice in The Netherlands are presented.

Recently, collaboration has been enhanced by advances in information and communication technology that have enabled information sharing among carriers. Information can be shared in two alternative ways. In a centralized collaboration scheme, a central decision maker redistributes customers and/or logistic assets among carriers. This decision maker may be a third party who acts in a non-partisan way or may be a large carrier that resorts to other carriers to manage all its orders and customers. In a decentralized collaboration scheme, carriers exchange their orders individually or in clusters. In this case, carriers cooperate at the same level trusting each other for the information shared. All the above considerations and approaches apply to both truckload or less-than-truckload carriers. 
In this paper, we focus on situations where collaboration is managed in a centralized way. We consider a set of carriers cooperating under the guidance of a central station that acts in a non-partisan way. Each carrier has a depot and a set of customers. Each customer is represented with an arc and its service generates a revenue. Each carrier identifies a subset of customers that it wants or needs to serve. These customers may be the most easily served, the most profitable or the most strategic ones. The remaining customers are defined as shared customers, that is customers that may be served by other carriers. A shared customer may end up being served by the carrier that decided to share it, when combined with customers shared by other carriers. Part of the revenue of a shared customer goes to the carrier that decided to share the customer and part goes to the carrier that actually serves it. We allow a shared customer not to be served by any carrier of the coalition. In this case the revenue is not collected by any carrier. This corresponds to the situation where the customer is not profitable for any carrier of the collaborating group and in a further phase a different and interested carrier will be searched. We assume that each carrier has one vehicle and that vehicle capacity is not relevant, that is the vehicles are uncapacitated.

The motivation for studying this problem comes from potential applications. In general, applications arise in private companies offering services which allow competition and collaboration, and where customers may be modelled as arcs of a network. As an example we mention home pick-up and delivery, including private mail and small packaging distribution, and taxi services. For example, the problem that we address can model a group of independent taxi drivers collaborating under the guidance of a central station.

We call the proposed problem, that may be seen as belonging to the class of arc routing problems with profits, Collaboration Uncapacitated Arc Routing Problem (CUARP). We study two different variants of the CUARP. In the first one the goal is the maximization of the total profit of the coalition of carriers, independently of the individual profit of each carrier. The second variant includes a lower bound on the individual profit of each carrier. This lower bound may represent the profit of the carrier in the case no collaboration is implemented. We formulate mixed integer programming models for the two variants of the problem and study their relations with well-known arc routing problems. We also look at the CUARP from a game theory perspective. As it is usual in arc routing problems, the proposed formulations have a number of connectivity constraints which is exponential in the number of customers. This leads us to study the separation problem for such constraints. We solve the formulations for the two proposed variants with a branch-and-cut algorithm and quantify the impact of collaboration. Starting from 118 benchmark instances for the Privatized Rural Postman problem, we generate a total of 971 instances, with 2 or 3 carriers and varying characteristics, such as different locations of the depots and different thresholds for the profit. We solve all instances within few seconds. On each instance we compare the optimal solution obtained in the case where no collaboration is allowed with the case where collaboration is allowed, and show that the profit of the coalition increases up to twice or even three times the profit achieved without collaboration. 
The rest of the paper is organized as follows. Section 2 introduces the relevant literature. The two variants of the CUARP are formally described and formulated in Section 3. Section 4 presents the theoretical results. In Section 5 we describe the separation procedure for the connectivity constraints that is used in the branch-and-cut algorithm. Data generation and computational experiments are described in Section 6. Finally, conclusions and future work are discussed in Section 7.

\section{Literature review}

The literature on collaboration in transportation can be divided in two streams, one on vertical and the other on horizontal collaboration. Vertical collaboration arises when shippers and customers collaborate to help each other optimize their objective, while horizontal collaboration takes place when shippers collaborate among them (and/or the same do customers) at the same logistic level. Ergun et al. [19] develop a collaboration model among shippers, involving only full truckload companies, to identify tours that minimize asset repositioning costs. The same authors discuss in [18] how to reduce truckload transportation cost through the identification of repeatable, dedicated continuous move tours using collaboration among carriers to reduce the need for repositioning and lowering costs. Mason et al. [28] focus on customer driven supply chain and freight management with the aim of studying if collaborative models for management transportation give optimized solutions.

Some authors addressed carrier collaboration from a perspective of costs and profits allocations, possibly within a game theory context. Figliozzi [20] proposes a setting in which a set of carriers, each with its own customers, has some incentive to submit all customers requests to a centralized collaborative decision making mechanism based on sequential second-price auction. Ozener et al. [30] focus, instead, on reducing costs through collaboration. Given a set of lanes carriers have to serve, their aim is to set up a process to exchange lanes either sharing or not sharing information about customers and/or side payments. Argawal and Ergun [1] study transportation networks that operate as an alliance among different carriers. They focus on formation of alliances and network design using both mathematical programming and game theory to investigate the mechanism that leads to an optimal collaborative strategy. In contrast to those studies, in our setting we deal with a network of carriers (regional or functional) that form a coalition to collaborate and we consider as a given fact that collaboration is better than competition, as pointed out in Argawal [2], Meyer [29], and Fugate [21].

Audy et al. [9] and Krajewska et al. [26] are case oriented papers. The former deals with the supply chain of the Canadian furniture industry, while the latter deals with more general coalitions among carriers. Both make use of game theory to allocate cost among companies, customers, carriers and coalitions. In particular, in [26] the authors also use the classic Shapley value to allocate costs among carriers and coalitions of carriers. In [23] various criteria are presented to allocate costs using classical game 
theory in a vehicle routing problem. Our perspective in this paper is quite different. While we do not focus on cost allocation among carriers, we study how to improve profits for the whole carriers network within the framework of a fixed collaboration agreement by stating our model as a prize-collecting arc routing problem with several carriers and depots.

Since the CUARP belongs to the class of arc routing problems with profits, we next recall some relevant literature related to this class. Christofides et al. [12] present a directed version of the Rural Postman Problem (RPP) [27], which is later generalized as the Directed Profitable RPP (DPRPP) in [7], where some arcs may not be served by paying a penalty for each of them. Aráoz et al. [6] propose the Privatized Rural Postman Problem (PRPP) whose objective is to find a tour maximizing the profit gained, starting and ending at a fixed depot. Different variants of this problem were proposed by several authors. We mention the Clustered Prize-Collecting Arc Routing Problem introduced by Aráoz et al. [4] and its windy version studied by Corberán et al. [13]. For a comprehensive survey on arc routing with profits we refer to Archetti et al. [8]. Differently from the above studies, we focus on a multi-depot model to optimize collaboration among carriers.

\section{Collaboration Uncapacitated Arc Routing Problem}

The CUARP can be stated as follows. We consider a set of carriers, each with one depot and one vehicle. We assume that the problem is uncapacitated and do not consider capacity constraints on vehicles. Customers are represented as arcs of a graph and are served when the vehicle traverses the corresponding arcs. Carriers reach a collaboration agreement, described in the following, under the guidance and surveillance of a third party central decision maker. The goal is to find one route for each carrier, in the framework of the collaboration agreement, such that the profit is maximized. The collaboration scheme that we study is the following. Each customer is associated with a specific carrier. Each carrier partitions its customers in two sets:

- customers the carrier must serve because of contractual obligations or other types of considerations, such as relevance or convenience;

- customers the carrier is willing to share with other carriers, because of a low level of geographical synergies with other customers or a low profitability.

The customers of the first type are called required and form the required set, whereas the customers of the second type are called shared and form the shared set. Required and shared customers are called demand customers. We will refer both to customers associated with (or assigned to) carriers and to carriers associated with (or assigned to) customers. We note that each customer is assigned to one carrier, whereas there are 
usually several customers assigned to one carrier. While required customers must be served by their associated carrier, shared customers can be served by any carrier. We allow a shared customer not be served by any carrier of the coalition. This corresponds to the situation where the customer is not profitable for any carrier and, in the later stage, a carrier that does not belong to the coalition will be searched.

Each customer, if served, will pay an amount of money to its associated carrier. For each shared customer the associated carrier will share part of this revenue with the carrier that will end up actually serving the customer. Carriers determine the side payment for each shared customer for the case it will be served by a different carrier.

Each customer can be served at most once and by only one carrier. Thus, the revenue is collected only the first time the corresponding arc is traversed, even if the arc is traversed more than once. If a shared customer is not served, a penalty is charged to the associated carrier. Every time an arc is traversed a cost is charged, independently of whether or not it corresponds to a demand arc.

We identify carriers with vehicles, depots, and routes, and assume that routes start and end at the same depot.

In order to state the CUARP formally, we first introduce some notation. Let $G=(V, A)$ be a strongly connected directed graph with vertex set $V=\{1, \ldots, n\}$ and $\operatorname{arc}$ set $A$. When needed, arcs will be denoted by their end-vertices $a=(u, v)$. A non-negative traversal cost $c_{a}$ is associated with each arc $a \in A$. The subset of demand arcs (customers) is denoted by $D \subset A$, and the subsets of required and shared customers by $R$ and $S$, respectively. We have $D=R \cup S$ and $R \cap S=\emptyset$. A non-negative value $r_{a}$ is associated with each demand arc $a \in D$, which represents the money offered by customer $a$ in exchange of service. Furthermore, a non-negative value $g_{a} \leq r_{a}$ is associated with each shared arc $a \in S$, which represents the side payment from the associated carrier to the carrier that provides the service to $a$. A positive value $\phi_{a}$ is also associated with each shared arc $a \in S$, which is the penalty that the associated carrier must pay for not serving customer $a$.

Let $L=\{1, \ldots, k\}$ be the index set for the carriers, each of them with a depot located at a vertex of the graph, denoted by $v^{l} \in V, l \in L$. We also use $V^{L}=\left\{v^{l} \mid l \in\right.$ $L\} \subset V$ to denote the set of all depots and $I^{l}=L \backslash\{l\}$, for $l \in L$. For each $l \in L$ we denote by $D^{l}$ the subset of demand customers associated with $l$, and by $R^{l}=D^{l} \cap R$ and $S^{l}=D^{l} \cap S$ its associated required and shared customers, respectively. Customers in $D^{l}, R^{l}$, and $S^{l}$ will be referred to as $l$-demand, l-required and $l$-shared customers, respectively. For $l \in L, D^{l}=R^{l} \bigcup S^{l}$ and $R^{l} \bigcap S^{l}=\emptyset$. We also have, $D=\bigcup_{l \in L} D^{l}$, $R=\bigcup_{l \in L} R^{l}$, and $S=\bigcup_{l \in L} S^{l}$. 
We use the following standard notation. For a nonempty proper subset $F \subseteq V$, $\gamma(F)=\{a=(u, v) \in A \mid u, v \in F\}, \quad$ set of arcs with both vertices in $F$, $\delta^{+}(F)=\{a=(u, v) \in A \mid(u \in F, v \notin F)\}$, set of arcs that start in $F$ and end out of it, $\delta^{-}(F)=\{a=(u, v) \in A \mid(u \notin F, v \in F)\}$, set of arcs that start out of $F$ and end in it.

Finally, for each $H \subseteq A$ we define $y(H)$ as $\sum_{a \in H} y_{a}$.

In the CUARP, we impose that for any carrier $l \in L$, each $l$-required arc $a \in R^{l}$ is served by carrier $l$. Instead, an $l$-shared arc $a \in S^{l}$ can be served either by carrier $l$ or by a different carrier $h \in I^{l}$, or not served at all. A customer $a \in D$ offers a non-negative amount of money $r_{a}$ in exchange of service. Carrier $l$ collects the revenue $r_{a}$ for each $l$-required arc $a \in R^{l}$ as well as for each served $l$-shared arc $a \in S^{l}$, even if it is served by a different carrier $h \in I^{l}$. If carrier $h$ serves an $l$-shared arc $a \in S^{l}$, it collects the non-negative side payment $g_{a}$ from carrier $l$. Therefore, the side payment $g_{a}$ is added to the profit of carrier $h$ and subtracted from the profit of carrier $l$. If an $l$-shared arc $a \in S^{l}$ is not served by any carrier, then the revenue $r_{a}$ is not collected by carrier $l$, and carrier $l$ gives no side payment $g_{a}$ to any other carrier. However, in this case carrier $l$ has to pay the penalty $\phi_{a}$. All routes start and end at the depot of their associated carrier. While carriers with a non-empty required set must certainly perform a route, it is possible for a carrier with empty required set to perform no route. If performed, the route of such a carrier will only serve shared arcs. Carrier $l$ pays a $\operatorname{cost} c_{a}$ each time arc $a \in A$ is traversed in its route. The total profit of carrier $l \in L$ is the difference between its total income and its total costs and side payments, including penalties. The aim of the CUARP is to maximize the total profit of the coalition of carriers.

\subsection{Formulations}

To formulate the CUARP we define the following two sets of decision variables, which identify the arcs that are served and traversed by each carrier $l \in L$.

For each $a \in A$, let

$$
\begin{gathered}
y_{a}^{l}= \begin{cases}1 & \text { if } a \text { is served by vehicle } l, \\
0 & \text { otherwise }\end{cases} \\
x_{a}^{l}=\text { number of times vehicle } l \text { traverses } a .
\end{gathered}
$$

Associated with a solution $\left(x_{a}^{l}, y_{a}^{l}\right)$ we define the following functions for each carrier 
$l \in L:$

$C_{1}^{l}=\sum_{a \in A} c_{a} x_{a}^{l}, \quad$ total traveling cost for carrier $l$,

$C_{2}^{l}=\sum_{a \in S^{l}} \phi_{a}\left(1-y_{a}^{l}\right), \quad$ total penalty payed by carrier $l$,

$C^{l}=C_{1}^{l}+C_{2}^{l}, \quad$ total cost for carrier $l$,

$P_{1}^{l}=\sum_{a \in D^{l}} r_{a} y_{a}^{l}+\sum_{a \in S^{l}}\left[\left(r_{a}-g_{a}\right) \sum_{i \in I^{l}} y_{a}^{i}\right]$, profit collected by carrier $l$ from $l$-demand customers,

$P_{2}^{l}=\sum_{i \in I^{l}}\left(\sum_{a \in S^{i}} g_{a} y_{a}^{l}\right), \quad$ total side payments collected by carrier $l$ from other carriers,

$P^{l}=P_{1}^{l}+P_{2}^{l}-C^{l}, \quad$ total profit of carrier $l$.

Furthermore, the set of constraints $(C)$ models the collaboration agreement among carriers:

$$
(C) \begin{cases}x^{l}\left(\delta^{+}(u)\right)=x^{l}\left(\delta^{-}(u)\right) & l \in L, u \in V \\ x^{l}\left(\delta^{+}\left(v^{l}\right)\right) \geq 1 & l \in L \text { with } R^{l} \neq \emptyset \\ x^{l}\left(\delta^{+}\left(v^{l}\right)\right) \geq y_{a}^{l} & l \in L \text { with } R^{l}=\emptyset, a \in S \\ x^{l}\left(\delta^{+}(F)\right) \geq y_{a}^{l} & l \in L, F \subset V \backslash\left\{v^{l}\right\}, a \in \gamma(F) \\ y_{a}^{l}=1, & a \in R^{l}, l \in L \\ \sum_{l \in L} y_{a}^{l} \leq 1 & a \in S \\ y_{a}^{l} \leq x_{a}^{l} & a \in A, l \in L .\end{cases}
$$

Flow-in flow-out constraints (1) guarantee the symmetry of the vertices, because the number of incoming arcs must be equal to the number of outgoing arcs. Constraints (2) and (3) guarantee that the carriers routes start from their depots. While (2) imposes a route to any carrier $l \in L$ with non-empty required set, constraints (3) only impose a route to carriers with empty required sets who serve some shared arc. Constraints (4) guarantee that the route of each carrier is connected. Given a subset $F \subset V \backslash\left\{v^{l}\right\}$, if an arc $a \in \gamma(F)$ is served, then some arc $b \in \delta^{+}(F)$ must be traversed at least once. Hence, each carrier travels a connected route because if it serves some arc from a subset of arcs which does not contain the depot then it has to leave the subset. Note, however, that Constraints (4) do not prevent subtours containing no served arc. Since such subtours produce no profit, they will never appear in any optimal solution. Constraints (4), together with constraints (1)-(3), also guarantee that each carrier route ends at its depot. Constraints (5) force carrier $l$ to serve all $l$-required arcs, whereas inequalities (6) ensure that $l$-shared arcs are served by at most one carrier. Finally, inequalities (7) impose that all arcs served by a given carrier are traversed by that carrier. 
We introduce now the mathematical programming formulation for the CUARP, where we maximize the total profit of the coalition of carriers:

$$
\begin{aligned}
z_{c}=\max & \sum_{l \in L} P^{l} \\
& (C) \\
& x_{a}^{l} \in \mathbb{Z}^{+}, \quad a \in A, l \in L ; \quad y_{a}^{l} \in\{0,1\}, \quad a \in D, l \in L .
\end{aligned}
$$

Remark 3.1 In the CUARP carriers exchange side payments corresponding to shared arcs served by carriers different from the ones they are assigned to. This means that if, for a given arc $a \in S^{l}$, some carrier $i \in I^{l}$ takes $g_{a}$ for serving $l$-shared arc $a$, then carrier $l$ takes $r_{a}-g_{a}$. Thus, the profit collected by all carriers is:

$$
\begin{gathered}
\sum_{l \in L}\left(P_{1}^{l}+P_{2}^{l}\right)-C^{l}=\sum_{l \in L}\left(\sum_{a \in D^{l}} r_{a} y_{a}^{l}+\sum_{a \in S^{l}}\left[\left(r_{a}-g_{a}\right) \sum_{i \in I^{l}} y_{a}^{i}\right]+\sum_{i \in I^{l}} \sum_{a \in S^{i}} g_{a} y_{a}^{l}\right)-\sum_{l \in L} C^{l}= \\
\sum_{l \in L}\left(\sum_{a \in D^{l}} r_{a} y_{a}^{l}+\sum_{a \in S^{l}} r_{a} \sum_{i \in I^{l}} y_{a}^{i}-C^{l}\right)
\end{gathered}
$$

where the last equality follows as the following two sums cancel out since:

$$
\sum_{l \in L} \sum_{a \in S^{l}} g_{a} \sum_{i \in I^{l}} y_{a}^{i}=\sum_{l \in L} \sum_{i \in I^{l}} \sum_{a \in S^{i}} g_{a} y_{a}^{l} .
$$

Hence, the objective function (8) can be reformulated as:

$$
\max \sum_{l \in L}\left(\sum_{a \in D^{l}} r_{a} y_{a}^{l}+\sum_{a \in S^{l}} r_{a} \sum_{i \in I^{l}} y_{a}^{i}-C^{l}\right) .
$$

Therefore, the optimal solution to a CUARP instance is independent of the side payments $g_{a}, a \in S$, since (11) does not depend on the side payments $g_{a}, a \in S$, and the domain (1)-(7) is independent of the side payments as well. Thus, we have that an optimal solution to a CUARP instance with side payments $g_{a}, a \in S$ is also optimal to a CUARP instance with side payments $g_{a}^{\prime}, a \in S$, if all other data remain unchanged.

In the CUARP we can force carriers not to collaborate by adding a constraint that prevents carrier $l$ from serving arcs outside its demand set:

$$
y_{a}^{i}=0, \quad a \in S^{l}, i \in I^{l} .
$$


We define the CUARP without collaboration, that we denote as $n$-CUARP, as the problem obtained by simply adding the above constraints to the CUARP formulation:

$$
\begin{aligned}
& z_{n c}=\max \sum_{l \in L} P^{l} \\
& \text { (C) } \\
& y_{a}^{i}=0 \\
& a \in S^{l}, i \in I^{l} \\
& x_{a}^{l} \in \mathbb{Z}^{+}, \quad a \in A, l \in L ; \quad y_{a}^{l} \in\{0,1\}, \quad a \in D, l \in L .
\end{aligned}
$$

Observe that the CUARP does not guarantee any profit balance among carriers, possibly limiting the interest for carriers to collaborate. Let us consider, for example, the case of a carrier that has an associated customer that generates little profit because it is not very conveniently located. If the carrier decides to share this customer, the customer might be end up being served by another carrier, for which it is more conveniently located. However, the carrier that decided to share it may simply loose the little profit of the shared customer without gaining anything. In the CUARP the largest carriers will tend to benefit from collaboration more than the small ones.

Below we introduce a variant of the CUARP, that we call the $t$-CUARP, in which a minimum profit threshold $t^{l}$ is guaranteed for any carrier $l \in L$. Each carrier may set the threshold to avoid reducing its profit because of collaboration. The threshold for carrier $l$ might be set to be its profit in the $n$-CUARP. The resulting formulation for the $t$-CUARP is:

$$
\begin{aligned}
& z_{t}=\max \sum_{l \in L} P^{l} \\
& \text { (C) } \\
& P^{l} \geq t^{l} \\
& x_{a}^{l} \in \mathbb{Z}^{+}, \quad a \in A, l \in L ; \quad y_{a}^{l} \in\{0,1\}, \quad a \in D, l \in L .
\end{aligned}
$$

In the $t$-CUARP we maximize the total profit of the carriers coalition (16) as long as the profit of each carrier is not smaller than its threshold (see (18)). In contrast to the CUARP, introducing profit thresholds in the $t$-CUARP gives to side payments $g_{a}$ a central role, since constraints (18) depend on their values. Indeed, it may now happen that a solution which is feasible for the CUARP is no longer feasible for the $t$-CUARP, because the amount of side payments from a carrier to the others may cause its profit to fall below the given threshold.

We illustrate the behaviour of the different models on an example.

Example 3.1 In Figure 1(a) a small instance is shown. We consider a graph with 4 


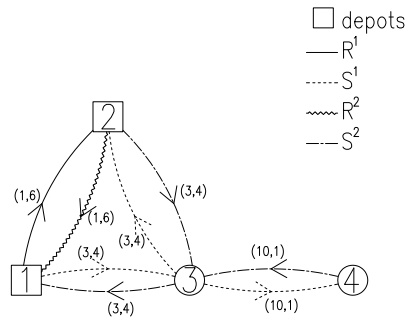

(a) Instance graph
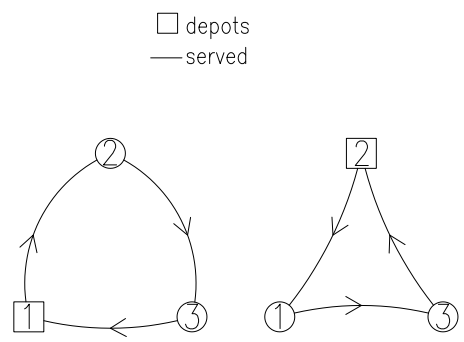

(c) CUARP solutions

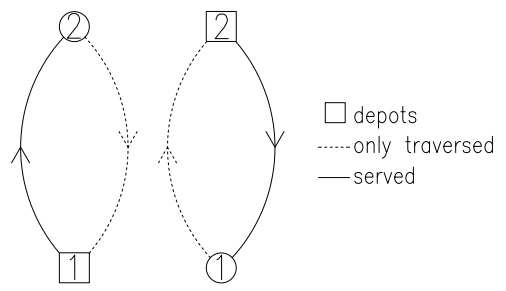

(b) $n$-CUARP solutions

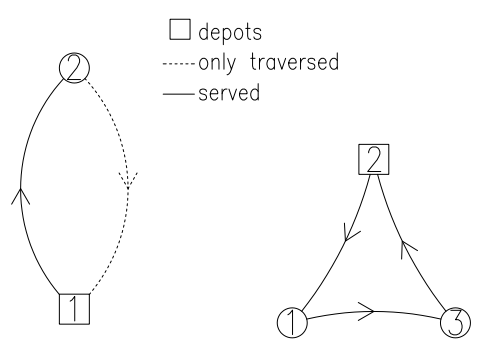

(d) $t$-CUARP solutions

Figure 1: Example

vertices $\{1,2,3,4\}$, whose arcs are partitioned as follows:

$$
\begin{array}{lr}
R^{1}=\{(1,2)\} & R^{2}=\{(2,1)\} \\
S^{1}=\{(1,3) ;(3,2) ;(3,4)\} & S^{2}=\{(2,3) ;(3,1) ;(4,3)\} .
\end{array}
$$

We have only two depots in 1 and 2, respectively. Penalties are set to 1 for each shared arc. Each arc in Figure 1(a) has a label with two numbers, the first one is the traversing cost and the second one is the profit for serving it. We set the following side payments for serving shared arcs as follows:

$$
g_{(2,3)}=g_{(3,1)}=g_{(4,3)}=g_{(3,4)}=1, \quad g_{(1,3)}=g_{(3,2)}=4 .
$$

In Figure 1(b) we represent the solutions of the CUARP without collaboration. Carrier 1 serves its required customer $(1,2)$ in the route $1-2-1$, with a profit of 4 , and pays the penalty for its unserved shared arcs $(1,3),(3,2)$ and $(3,4)$ with a total penalty of 3 . Carrier 2 serves its required customer $(2,1)$ in the route $2-1-2$, with a profit of 4 , and pays the penalty for its unserved shared arcs $(2,3),(3,1)$ and $(4,3)$ with a total penalty of 3. The total profit for the coalition of carriers 1 and 2 is $2 . \quad$ In Figure 1(c) the solutions of the CUARP are shown. The route of carrier 1 is $1-2-3-1$. Its profit is $P^{1}=0$. Similarly, the route of carrier 2 is $2-1-3-2$, with a profit $P^{2}=12$. Thus, the collaboration between the carriers leads to a total profit of 12 , with a profit increase of $83.33 \%$ with respect to the solution of the CUARP without collaboration. Note that the profit is totally gained by carrier 2 , while carrier 1 has a null profit $\left(P^{1}=0, P^{2}=12\right)$. This allocation of the profit in the coalition of carriers is due to the side payments exchanges between carriers. According to this, carrier 1 has no incentive to collaborate 
with carrier 2, as this would result in a profit decrease for carrier 1 . Observe that the CUARP solution shown in Figure 1(c) is not feasible for the $t$-CUARP instance with profit thresholds set to the individual profits of the $n$-CUARP (i.e. $t^{1}=t^{2}=1$ ). Figure 1(d) shows the optimal routes for this $t$-CUARP instance, which are 1-2-1 and 2-1-3-2, for carriers 1 and 2, respectively. As in the $n$-CUARP solution, carrier 1 only serves its required arc but no shared arc. On the contrary, carrier 2 serves not only its required arc, but also the 1-shared arcs $(1,3)$ and $(3,2)$, as in the CUARP solution. Less shared arcs are served with respect to the CUARP. Now, the profit of carriers 1 and 2 are $P^{1}=3$ and $P^{2}=4$, respectively. Hence, the total profit of the two carriers is equal to 7 which means a profit improvement of $71.43 \%$ with respect to the $n$-CUARP and a decrease of $41.66 \%$ with respect to the CUARP solution. However, the profit coming from collaboration is shared between carriers in a fairer way because of the profit thresholds. In Table 1 we summarize profit sharing for the different models in the Example 3.1.

Table 1: Summary of Example 3.1

\begin{tabular}{|r|r|r|r|}
\hline carrier & $n$-CUARP & CUARP & $t$-CUARP \\
\hline 1 & 1 & 0 & 3 \\
\hline 2 & 1 & 12 & 4 \\
\hline
\end{tabular}

It may be expected that, in the $t$-CUARP, a higher value of the side payments results in an increase in the number of arcs served by the coalition. In the following example, we illustrate a counter-intuitive behaviour of the model.

Example 3.2 In this example we illustrate the difference between the CUARP and the $t$-CUARP and the effect of side payments in the $t$-CUARP. Consider a CUARP instance defined on the same directed graph of Example 3.1 with the same two carriers and the same sets of required and shared arcs for each carrier. Suppose $\phi_{a}=0$ for all $a \in D$, with the following values for the profits and costs: $r_{12}=r_{21}=6 ; r_{13}=r_{32}=4$; $r_{23}=r_{31}=r_{34}=r_{43}=1 ; c_{12}=c_{21}=1 ; c_{13}=c_{32}=c_{23}=c_{31}=3 ; c_{34}=c_{43}=10$. In the optimal $n$-CUARP solution carrier 1 serves its required customer $(1,2)$ in the route 1-2-1, with a profit of 4 . Similarly, carrier 2 serves its required customer $(2,1)$ in the route $2-1-2$, with a profit of 4 . When collaboration is allowed, in the optimal CUARP solution the routes for carriers 1 and 2 become 1-2-1 and 2-1-3-2, respectively. If we set $g_{a}=\beta r_{a}$ for all $a \in D$ with $\beta=1$, we have the following distribution of profits: Carrier 1 gains $P^{1}=4$ and Carrier $2 P^{2}=7$. If we set $\beta=0.5$, profits become $P^{1}=8$ and $P^{2}=3$. When we consider the $t$-CUARP with profit thresholds set on the individual profits of the $n$-CUARP, with $t^{4}=t^{2}=4$, we observe that the solution with $\beta=1$ is still feasible, while that with $\beta=0.5$ is no longer feasible for the $t$-CUARP. For the case $\beta=0.5$, the optimal $t$-CUARP solution keeps unchanged the route of carrier 2 and assigns to carrier 1 the route 1-2-3-1. Hence, the total profit of carrier 1 is $P^{1}=4$ and that of carrier 2 is $P^{2}=4$. Comparing the $t$-CUARP solutions with $\beta=1$ and $\beta=0.5$ we note that in the former case 2 shared arcs are served while in the latter 4 shared arcs are served. Counter-intuitively, the percentage 
of shared arcs increases when decreasing $\beta$. In Table 2 we summarize profit sharing for the different models in the Example 3.2.

Table 2: Summary of Example 3.2

\begin{tabular}{|r|r|r|r|r|r|}
\hline & $\beta$ independent & \multicolumn{2}{|c|}{$\beta=1$} & \multicolumn{2}{|c|}{$\beta=0.5$} \\
\hline carrier & $n$-CUARP & CUARP & $t$-CUARP & CUARP & $t$-CUARP \\
\hline 1 & 4 & 4 & 4 & 8 & 4 \\
\hline 2 & 4 & 7 & 7 & 3 & 4 \\
\hline
\end{tabular}

\section{Theoretical results}

In this section we present some theoretical results for the CUARP.

\subsection{Reduction to other problems and complexity}

We analyze two particular cases of the CUARP with one single carrier:

- If the shared set $S$ is empty, then $D=R$ and, thus, the single carrier CUARP reduces to the Directed RPP (DRPP). Since the DRPP is known to be NP-Hard (see Lenstra and Rinnooy Kan [27]), also the CUARP is.

- If the required set $R$ is empty, then $D=S$ and, thus, the single carrier CUARP reduces to the DPRPP. Since the DPRPP is NP-hard (see Archetti et al. [7]), this is an alternative proof that CUARP is NP-Hard.

Thus, we can reduce the single carrier CUARP to other problems by changing the size of the shared and required sets. At one extreme, with no shared arcs, we have the DRPP, whereas on the other one, with no required arc, we have the DPRPP.

\subsection{Impact of collaboration on profit}

Remark 4.1 Let $\mathcal{I}$ be a CUARP instance and $z_{c}$, $z_{n c}$ be the CUARP optimal value and the $n$-CUARP optimal value over $\mathcal{I}$, respectively. Then, $z_{n c} \leq z_{c}$.

This result holds trivially, since any feasible solution to the $n$-CUARP is a CUARP feasible solution. 
Remark 4.2 Let $\mathcal{I}^{1}, \mathcal{I}^{2}$ be two CUARP instances and $z_{c_{1}}$, $z_{c_{2}}$ their CUARP optimal values. Suppose these instances differ only because the shared set of the first one is contained in the shared set of the second one and vice versa for the required sets. Then, $z_{c_{1}} \leq z_{c_{2}}$.

This result holds trivially, since any feasible solution for $\mathcal{I}^{1}$ is a feasible solution for $\mathcal{I}^{2}$. We may say that the attractiveness of collaboration increases by increasing the shared arcs or by decreasing the required arcs.

Remark 4.3 Let $\mathcal{I}$ be a CUARP instance, and let $z_{t}, z_{n c}$ be, respectively, the optimal $t$-CUARP and $n$-CUARP values over $\mathcal{I}$, when the $t$-CUARP thresholds $t^{l}, l \in L$, are set to the carriers profits of the $n-C U A R P$. Then, $z_{n c} \leq z_{t}$.

The above result follows, since for instance $\mathcal{I}$ the optimal solution to the $n$-CUARP is also feasible for the $t$-CUARP with the given threshold values.

Proposition 4.1 Let $z_{c}$ and $z_{n c}$ denote the optimal values of the CUARP and the $n$ CUARP over a given instance $\mathcal{I}$, respectively. There exists no finite upper bound for the profit increase ratio $\frac{z_{c}}{z_{n c}}$.

Proof: Consider a CUARP instance defined on the same directed graph of Example 3.1 with the same two carriers and the same sets of required and shared arcs for each carrier. Let $K>0$ and $\varepsilon>0$ be two given values and suppose $c_{a}=K$, for all $a \in A$, and $\phi_{a}=\varepsilon, g_{a}=K$, for all $a \in D$. Let us also suppose the profits are the following: $r_{12}=r_{21}=2 K+4 \varepsilon ; r_{13}=r_{31}=r_{23}=r_{32}=2 K-\varepsilon ; r_{34}=r_{43}=K$.

For this instance, in the optimal $n$-CUARP solution carrier 1 serves its required customer $(1,2)$ in the route $1-2-1$, with a profit of $4 \varepsilon$, and pays the penalty for all its unserved shared arcs $(1,3),(3,2)$ and $(3,4)$ with a total penalty of $3 \varepsilon$. Similarly, in the optimal $n$-CUARP solution, carrier 2 serves its required customer $(2,1)$ in the route $2-1-2$, with a profit of $4 \varepsilon$, and pays the penalty for all its unserved shared arcs $(2,3)$, $(3,1)$ and $(4,3)$ with a total penalty of $3 \varepsilon$. Hence, for this instance $z_{n c}=2 \varepsilon$.

When collaboration is applied, in the optimal CUARP solution carrier 1 serves its required customer $(1,2)$ and the 2 -shared customers $(2,3)$ and $(3,1)$ in route $1-2-3-1$, whereas carrier 2 serves its required customer $(2,1)$ and the 1 -shared customers $(1,3)$ and $(3,2)$ in route $2-1-3-2$. Demand customers $(3,4)$ and $(4,3)$ remain unserved. The profit of both carrier 1 and carrier 2 is, thus, $(K+4 \varepsilon)+(K-K)+(K-K)+(2 K-$ $\varepsilon-K)+(2 K-\varepsilon-K)-\varepsilon=3 K+\varepsilon$. Hence, for this instance $z_{c}=6 K+2 \varepsilon$.

Therefore, the profit increase ratio is $\frac{z_{c}}{z_{n c}}=\frac{6 K+2 \varepsilon}{2 \varepsilon}$, which tends to $\infty$ either when $K \rightarrow \infty$ or when $\varepsilon \rightarrow 0$. 
Observe that the optimal solution of the CUARP instance built in the above proof is also feasible for the $t$-CUARP when the threshold of each carrier is set to its profit without collaboration, i.e., $t^{1}=t^{2}=\varepsilon$. Therefore the following result also holds:

Corollary 4.2 Let $z_{t}$ and $z_{n c}$ denote the optimal values of the $t$-CUARP and the $n$ $C U A R P$ over a given instance $\mathcal{I}$, respectively. There exists no finite upper bound for the profit increase ratio $\frac{z_{t}}{z_{n c}}$.

\subsection{Game theory results}

We introduce here some basic concepts and definitions of cooperative game theory (see Driessen [15] for a comprehensive survey), and relate them to the CUARP. A cooperative game consists of a finite number $n$ of players and a characteristic function. Players can form different coalitions in order to achieve a better result in the game. The coalition of all players is called grand coalition. The characteristic function $v: 2^{n} \rightarrow \mathbb{R}$ is a function from the set of all possible coalitions of players to a set of payments such that $v(\emptyset)=0$. Each coalition is associated with a payment and each player has its own share of payment. In general, there are many different payments allocations suitable as solutions for a cooperative game. Hence, a key concept in cooperative game theory is the core, which is formed by those payments allocations $y_{1}, \ldots y_{n}$ that satisfy the following conditions:

$$
\begin{aligned}
& \sum_{j \in S} y_{j} \geq V(S), \text { with } S \subset N, \\
& \sum_{j \in N} y_{j}=V(N) .
\end{aligned}
$$

The former conditions prevent players from colluding to form a subcoalition in order to gain more. In the case $S=\{j\}$, the condition ensures that each player receives at least what he could get on his own, and is called individual rationality condition. The latter condition implies that the allocations $y_{1}, \ldots y_{n}$ split the total value gained by the grand coalition. This condition is called budget balance or efficiency condition. Given the above two conditions, no player has an advantage by leaving the grand coalition and the profit allocation is called stable.

We consider now a cooperative game based on the CUARP, using its mixed integer programming formulation as characteristic function, as suggested by Gothe et al. in [23]. Carriers play the role of game players. The CUARP and the $t$-CUARP allocate profit to the players. Both profit allocations fulfill the budget balance/efficiency condition because the sum of the profits of the carriers equals the maximum attainable profit of the whole coalition. However, the CUARP breaks the individual rationality condition because it may happen that a carrier gains more on its own without collaborating, as in Example 3.1. On the other hand, the $t$-CUARP with the $n$-CUARP profits 
as thresholds fulfills the individual rationality condition. Hence, the solution of the 2 carrier $t$-CUARP belongs to the core of the game when we set the $n$-CUARP profits as thresholds. In this case, the core is always non empty if the instance is feasible. In contrast, we cannot assure that the solutions of the $t$-CUARP with more than 2 carriers or with general thresholds belong to the core of the game. Even if the thresholds are set to the value of individual profit without collaboration, 2 or more carriers may collude to achieve a greater profit than that of the grand coalition. We might ensure that the $t$-CUARP profit allocation belongs to the core of the game, by adding a new set of constraints to the $t$-CUARP, imposing for each subset of carriers a minimum profit of at least its CUARP profit. However, adding this new set of constraints may cause the instance to become infeasible and the core of the cooperative game be empty. In a set of experiments this is what happened. Either the core was empty or the solution was identical to the solution without collaboration. Hence, we solved the $t$-CUARP adding only individual thresholds which are those that directly matter to the carriers.

\section{The branch-and-cut algorithm}

Inequalities (4) impose the connectivity of the route associated with each carrier with its depot. As the number of such constraints is exponential in the number of vertices in the input graph, $|V|$, we use a separation procedure that allows us to incorporate them in the formulation only when needed. Next, we describe the separation algorithm that we used for the exact solution of the CUARP and of the $t$-CUARP. The separation procedure uses as input vectors $\hat{x}=\left(\hat{x}^{l}\right)_{l \in L} \in R^{|A| \times|L|}$ and $\hat{y}=\left(\hat{y}^{l}\right)_{l \in L} \in R^{|A| \times|L|}$ satisfying constraints (1)-(3) and (5)-(7). The output of the algorithm will indicate whether or not there exists some inequality (4) violated by $\hat{x}$ and $\hat{y}$. In this case the algorithm will return one such inequality, i.e. the index of a carrier $l \in L$, a set $F \subset V \backslash\left\{v^{l}\right\}$ and an arc $a \in \gamma(F)$ such that the corresponding constraint (4) is violated by $\hat{x}$ and $\hat{y}$.

For each carrier $l \in L$, let $G^{l}\left(\hat{x}^{l}\right)=\left(V^{\hat{x}^{l}}, A^{\hat{x}^{l}}\right)$ denote the support graph of $\hat{x}^{l}$, with $A^{\hat{x}^{l}}=\left\{a \in A \mid \hat{x}_{a}^{l}>0\right\}$, and $V^{\hat{x}^{l}}=V\left(A^{\hat{x}^{l}}\right)$ the subset of vertices incident with some arc of $A^{\hat{x}^{l}}$. To separate inequalities (4), associated with a given carrier $l \in L$, we consider the support graph $G^{l}\left(\hat{x}^{l}\right)$. If $G\left(\hat{x}^{l}\right)$ is not connected, then each connected component with vertex set $C \subseteq V^{\hat{x}^{l}} \backslash\left\{v^{l}\right\}$ such that $\hat{y}(\gamma(C))>0$ defines a violated constraint (4) for carrier $l$, since $\hat{x}^{l}\left(\delta^{+}(C)\right)=0$ and $\hat{y}_{a}^{l}>0$, for some $a \in \gamma(C)$. If $G\left(\hat{x}^{l}\right)$ is connected, we compute the tree of min-cuts $T^{l}(\hat{x})$ relative to the capacities vector $\hat{x}^{l}$ (see, for instance, [22, 24]). Then, we use an adaptation of the algorithm of Belenguer and Benavent [10]. For each min-cut $\delta^{+}(F), v^{l} \notin F$, represented in $T^{l}(\hat{x})$, we identify the arc $\hat{a} \in \gamma(F)$ of maximum value, i.e. $\hat{a} \in \arg \max \left\{\hat{y}_{a}^{l} \mid a \in \gamma(F)\right\}$. Now, if $\hat{x}^{l}\left(\delta^{+}(F)\right)<\hat{y}_{\hat{a}}^{l}$, the connectivity constraint (4) associated with $l, F$ and $\hat{a}$ is violated by $\hat{x}^{l}$ and $\hat{y}^{l}$. The above separation is exact and similar to the procedure used by other authors to separate connectivity constraints similar to (4) for other arc routing problems $[11,3,4,13]$. 
The computational complexity of the above algorithm is dominated by that of the algorithm to obtain the min-cut tree associated with each carrier, which is $\mathcal{O}\left(|V|^{4}\right)$ as pointed out in [22] and in [24].

\section{Data generation and computational results}

We present in this section the numerical results obtained on a series of computational experiments. Programs were coded in Java using CPLEX 12.5 library (64 bit) for the solution of the mixed integer problems. Default parameters were used. All tests were run on a HP Z400 Workstation, 64 bit, $3.33 \mathrm{GHz}, 12.0$ RAM. Since there are no available CUARP benchmark instances, we generated instances from the 118 PRPP benchmark instances used in [5]. These PRPP instances were derived from well-known RPP instances, which are divided in five groups. The first group contains two data sets, A and B, obtained from the Albaida Spain Graph (see Corberán and Sanchis [14]). The second group contains the 24 instances (labeled P) of Christofides et al. [12]. The last three groups contain instances from Hertz et al. [25]: 36 instances with vertices of degree 4 and RPP disconnected required edge sets (labeled D), 36 grid instances (labeled G), and 20 randomly generated instances (labeled R). Below we explain how the remaining data of the instances were defined. First, the original undirected graph is transformed in a directed graph in the following way.

- All arcs are defined from edges of the original graph (see [5]).

- Each original edge is transformed in two arcs with probability 0.1, and in one single arc with probability 0.9 . In the latter case, the direction of the arc is randomly chosen with equal probability.

- Arcs inherit their costs from the original edges. When two arcs are generated from the same edge, both arcs have the same cost.

- If needed, when all original edges have been considered, additional arcs are defined to guarantee that the resulting graph is strongly connected.

- For each pair of vertices, $u, v \in V$, for which the directed graph defined earlier contains no path from $u$ to $v$, we define a non-demand arc $(u, v)$ and assign to it the cost of the shortest path in the undirected graph connecting $u$ and $v$.

- Demand arcs are selected starting from the required edges of [5], as follows:

- If the original edge is a demand edge that has been transformed in two arcs, both transformed arcs become demand arcs with probability 0.15 . Otherwise, one transformed arc is randomly selected as demand arc while the other one becomes non-demand. 
- If the original edge is a demand edge that has been transformed in one single arc, the transformed arc becomes a demand arc.

- If the original edge is non-demand the transformed arc(s) is(are) non-demand.

- The profit of each demand arc $a \in D$ is defined as $r_{a}=2 b_{e}$, where $b_{e}$ is the profit of the undirected edge in the corresponding PRPP instance [5]. If $e$ is a required edge of the RPP instance, $b_{e}$ is a number randomly generated from an integer uniform distribution in the range $\left[c_{e}, 3 c_{e}\right]$. Otherwise, $b_{e}=0$.

- The side payment of each demand arc $a \in D, g_{a}$, is set to a fraction $\beta$ of its profit, i.e., $g_{a}=\beta r_{a}$. For each instance, we use two values for $\beta$, namely 0.5 and 1.

To define the demand sets, we denote by $P_{u v}$ the shortest path from $u$ to $v$ in the directed graph and by $c\left(P_{u v}\right)$ its cost. Then, we define the following auxiliary set for each carrier $l \in L$ :

$$
\bar{D}^{l}=\left\{a \in D \mid w_{a} \leq 0.75 \wedge c\left(P_{v^{l} u}\right) \leq c\left(P_{v^{i} u}\right), \quad \text { for all } i \in I^{l}\right\},
$$

where $u$ denotes the end-vertex of arc $a$ closest to $v^{l}$ and $w_{a}$ a random number in $[0,1]$ associated with arc $a$. Since the sets $\bar{D}^{l}$ are not necessarily disjoint for different carriers the demand sets are finally defined as

$$
D^{1}=\bar{D}^{1}, \quad D^{l}=\bar{D}^{l} \backslash\left(\bigcup_{k=1}^{l-1} \overline{D^{k}}\right), l \in\{2, \ldots,|L|\}
$$

Broadly speaking, we assign demand arcs to the carrier with smallest distance from/to its depot with a probability of $75 \%$. Moreover, if through this procedure a required arc belongs to more than one $\bar{D}^{l}$ set, then it is assigned to the required set of the smallest index carrier, among the ones whose $\bar{D}^{l}$ sets contain the arc.

To divide each carrier demand set into shared and required sets, firstly, we list all demand arcs for each carrier according to their distance from the depot. Then, the less distant arcs form the required set while the remaining ones the shared set. Shared and required sets are defined to ensure that at least a percentage $p$ of the demand arcs are shared. We fix $p$ equal to $50 \%$. We denote with $M$ the vertex most distant from vertex 1 , and with $m$ the vertex whose distance from 1 is the median of all distances from 1 to $v \in V$.

We set the same penalty value for all the shared arcs associated with the same carrier. For each carrier $l \in L$, this penalty is set to the average profit loss per unserved $l$-shared arc. That is, for $l \in L, a \in S^{l}$,

$$
\phi_{a}= \begin{cases}\max \left\{0,\left\lfloor\sum_{a \in S^{l}} \frac{r_{a}-c_{a}}{\left|S^{l}\right|}\right\rfloor\right\} & \text { if } S^{l} \text { is not empty } \\ 0 & \text { otherwise. }\end{cases}
$$


The minimum threshold values $t^{l}, l \in L$, are given by the profits of the $n$-CUARP. The instances are available at the following link: http://or-brescia.unibs.it/ instances.

Table 3 summarizes information on the instances, which have been grouped according to their characteristics and to their sizes. These instances were generated with 2 depots located at $v^{1}=1$ and $v^{2}=M$. Columns under \#inst. and \#vertices give, respectively, the number of instances in the group and the number of vertices of the instances in the group. Columns under $\left|R^{1}\right|$ and $\left|R^{2}\right|$ give the number of 1-required and 2-required arcs, respectively. Similarly, columns under $\left|S^{1}\right|$ and $\left|S^{2}\right|$ give the number of 1-shared and 2-shared arcs.

Table 3: Instance summary

\begin{tabular}{|c|c|c|c|c|c|c|}
\hline & \#inst. & \#vertices & $\left|R^{1}\right|$ & $\left|R^{2}\right|$ & $\left|S^{1}\right|$ & $\left|S^{2}\right|$ \\
\hline AA & 1 & 102 & 48 & 34 & 48 & 35 \\
\hline AB & 1 & 90 & 50 & 23 & 50 & 23 \\
\hline $\mathrm{P}$ & 24 & $7-50$ & $0-48$ & $2-46$ & $1-48$ & $3-47$ \\
\hline D16 & 9 & 16 & $5-8$ & $7-10$ & $5-8$ & $8-11$ \\
\hline D36 & 9 & 36 & $15-21$ & $16-21$ & $15-21$ & $17-21$ \\
\hline D64 & 9 & 64 & $32-41$ & $20-32$ & $33-42$ & $21-33$ \\
\hline D100 & 9 & 100 & $48-67$ & $32-50$ & $49-67$ & $33-50$ \\
\hline G16 & 9 & 16 & $0-3$ & $0-3$ & $1-4$ & $1-3$ \\
\hline G36 & 9 & 36 & $1-9$ & $3-11$ & $1-9$ & $3-12$ \\
\hline G64 & 9 & 64 & $2-13$ & $6-21$ & $3-14$ & $6-22$ \\
\hline G100 & 9 & 100 & $8-29$ & $11-32$ & $8-29$ & $12-33$ \\
\hline $\mathrm{R} 20$ & 5 & 20 & $10-23$ & $5-15$ & $10-24$ & $6-16$ \\
\hline R30 & 5 & 30 & $19-37$ & $14-25$ & $19-37$ & $14-26$ \\
\hline $\mathrm{R} 40$ & 5 & 40 & $25-56$ & $16-47$ & $26-57$ & $17-48$ \\
\hline R50 & 5 & 50 & $33-57$ & $26-56$ & $33-58$ & $26-56$ \\
\hline
\end{tabular}

Table 4 summarizes the information on the values of the parameters that have been used and the number of tested instances in each case. The complete set of instances consists of 971 instances.

Table 4: Number of instances for each combination of parameters

\begin{tabular}{|l|c|c|l|l|l|}
\hline \multirow{2}{*}{ Location of depots } & \multirow{2}{*}{ CUARP } & \multirow{2}{*}{$n$-CUARP } & \multirow{2}{*}{$\begin{array}{c}\text { CUARP with } \\
\text { different proportions } \\
\text { of shared/required arcs }\end{array}$} & \multicolumn{2}{|c|}{$t$-CUARP } \\
\cline { 5 - 7 } & & & & 0.5 & 1 \\
\hline$v^{1}=1, v^{2}=M$ & 118 & 118 & 27 (G16) & 118 & 118 \\
$v^{1}=1, v^{2}=m$ & 118 & 118 & & & \\
$v^{1}=1, v^{2}=m, v^{3}=M$ & 118 & 118 & & & \\
\hline
\end{tabular}

A first set of experiments was run on the set of instances with two carriers, i.e. $L=\{1,2\}$, with depots $v^{1}$ and $v^{2}$ located at vertices 1 and $M$, respectively. The results for the CUARP are summarized in Table 5. Columns under CUARP and $n$ CUARP give the average net profit of carriers for the CUARP and the $n$-CUARP, respectively. Columns under \#S1 and \#S2 give the average number of shared arcs served by each carrier, while the column under \%serv. gives the average percentage of shared arcs served in total $\left(100 \frac{\# S 1+\# S 2}{\left|S^{1}\right|+S^{1} \mid}\right)$. Columns under \#S12 and \#S21 give, respectively, the average number of 1-shared arcs which are served by carrier 2, and vice versa. The column under \%exc. gives the average percentage of arcs exchanged between carriers (i.e. the average percentage of shared arcs that are served by a carrier different from the one they are assigned to, $\left.100 \frac{\# S 12+\# S 21}{\# S 1+\# S 2}\right)$. Column under increase gives the average percentage profit increase due to collaboration $\left(100\left|\frac{z_{c}-z_{n c}}{z_{c}}\right|\right)$. Finally, the last column under time gives the average computing times in seconds. 
The results of Table 5 illustrate the positive effect of the carriers collaboration. The average percentages \%serv. and \%exc. range in [67.0, 100.0] and in [41.0, 76.7], respectively. This means that there is a reasonable percentage of shared arcs served and exchanged between carriers. The average percentage profit increase of CUARP with respect to $n$-CUARP ranges in $[0.7,41.1]$. In general, the average CPU time required to solve small instances, such as those of P, D16, D36, G16, R20, R30, R40 groups, is less than 1 second. Solving the remaining instances required a little bit more computational effort, which pushed the CPU time up to a max of 158.0 seconds. The average CPU time is equal to 8.3 seconds. In Tables 6 and 7 we summarize the results for the $t$-CUARP, with $\beta=0.5$ and $\beta=1$, respectively. We tested separately the instances with these two different values of $\beta$, which affect the side payments and, thus, the profit threshold constraints. In these tables we have the additional columns increase 1 and increase 2 that show the profit increase for carrier 1 and 2. Comparing the results in Tables 6 and 7 with those in Table 5 we note that the average profit increase due to collaboration is smaller in $t$-CUARP than in CUARP. Instead, the average percentage \%serv. may even increase as, for example, for the instances in the D100 group in both cases with $\beta=0.5$ or $\beta=1$. The CPU times for the $t$-CUARP are comparable to those of the CUARP. We note that in some groups of instances the $t$-CUARP value is smaller when $\beta=1$ than when $\beta=0.5$. For instance, in $\mathrm{P} \%$ serv. increases from 90.5 (for $\beta=0.5$ ) to 91.1 (for $\beta=1$ ). Still the value of CUARP is greater for $\beta=0.5$ (392.8) than for $\beta=1$ (379.2). This $t$-CUARP behavior was explained in Remark 3.1 and illustrated in Example 3.2. Table 8 shows the characteristics of the instances that are obtained when displacing the depot of the second carrier $\left(v^{2}\right)$ from vertex $M$ to vertex $m$. Due to how sets $S^{l}$ and $R^{l}$ are defined when generating the instances, by displacing $v^{2}$ the average number of shared arcs increases and the average number of required arcs decreases. Table 9 summarizes the results with this new set of instances. Obviously, results in Table 9 cannot be compared with those in the previous tables, since we deal with completely different instances. The CPU times required to solve these new instances are comparable to those required for solving the instances in Table 3. Below we describe the results obtained in the experiments with CUARP and 3 depots, located in vertices $1, m$ and $M$, respectively. We have been able to solve all 118 instances. In Tables 10 and 11 we introduced additional columns to deal with 3 depots. In particular, columns under $\left|R^{3}\right|,\left|S^{3}\right|$ of Table 10 give the number of 3-required and 3-shared arcs, respectively. In Table 11 columns under \#S and \#Sexc give, respectively, the average number of shared arcs served and the average number of shared arcs exchanged. In Table 11 we note that the range of the average percentage of served shared arcs (\%serv.) and the average percentage of exchanged shared arcs and (\%exc.) is $[35.0,58.5]$ and $[26.4,46.5]$, respectively. Moreover, the average percentage profit increase ranges in $[0.0,16.8]$. However, comparing these results with those for the CUARP with 2 depots given in Table 5, we note that increasing the number of carriers from 2 to 3 does not necessarily increase the profit. As an example, for the G16 instances, when adding a new depot, increase decreases from $21.5 \%$ to $16.8 \%$. The computing times to solve instances with 3 carriers are similar to those required for the instances with 2 carriers. Finally, in order to test the behaviour of the model with different proportions of shared/required 
sets, some additional computational experiments were run. For these experiments we used the instances in G16 with depot located in 1 and in $M$. Firstly, we restricted the shared sets ensuring that at least $25 \%$ of the demand arcs belong to them. Then we enlarge them, ensuring that at least $75 \%$ of the demand arcs to be shared. Finally, we set them all as shared arcs. We performed 27 additional computational experiments. In Table 12 we show the profits for each case and each instance. Under columns $n$ CUARP, CUARP-25, CUARP, CUARP-75, CUARP-100 we give the optimal values for $n$-CUARP, CUARP with $25 \%$ of arcs shared, CUARP, CUARP with $75 \%$ and $100 \%$ of arcs shared, respectively. We note that profit does not decrease when we increase the number of arcs in the shared sets. This is consistent with Remark 4.2. We can conclude that the attractiveness of collaboration increases when we increase the number of shared arcs.

Table 5: Results for CUARP

\begin{tabular}{|c|c|c|c|c|c|c|c|c|c|c|}
\hline & n-CUARP & CUARP & $\# S 1$ & $\# S 2$ & $\# S 12$ & $\# S 21$ & \%serv. & \%exc. & increase & time \\
\hline$\overline{\mathrm{AA}}$ & 14442.0 & 24509.0 & 41.0 & 41.0 & 28.0 & 34.0 & 98.8 & 75.6 & 41.1 & 46.2 \\
\hline $\mathrm{AB}$ & 1411.0 & 17911.0 & 26.0 & 47.0 & 16.0 & 40.0 & 100.0 & 76.7 & 92.1 & 21.2 \\
\hline $\mathrm{P}$ & 379.2 & 392.9 & 13.4 & 12.9 & 8.3 & 7.6 & 90.5 & 54.7 & 4.0 & 0.4 \\
\hline D16 & 1211.8 & 1220.9 & 5.9 & 7.0 & 3.8 & 3.4 & 79.5 & 56.1 & 0.8 & 0.1 \\
\hline D36 & 2443.9 & 2460.6 & 17.1 & 15.9 & 9.1 & 8.3 & 90.8 & 52.7 & 0.7 & 0.4 \\
\hline D64 & 3582.6 & 3616.6 & 33.3 & 27.4 & 15.6 & 17.2 & 94.3 & 53.8 & 1.1 & 2.8 \\
\hline D100 & 4810.6 & 4873.2 & 42.7 & 54.0 & 22.4 & 35.8 & 97.5 & 60.2 & 1.5 & 14.6 \\
\hline G16 & 10.3 & 13.0 & 1.2 & 2.7 & 0.6 & 1.1 & 94.4 & 42.5 & 21.5 & 0.1 \\
\hline G36 & 42.8 & 51.0 & 4.8 & 8.0 & 2.3 & 3.0 & 100.0 & 41.0 & 16.0 & 1.4 \\
\hline G64 & 87.6 & 103.7 & 13.0 & 11.3 & 8.2 & 5.3 & 100.0 & 54.1 & 15.9 & 9.7 \\
\hline G100 & 139.9 & 170.8 & 21.9 & 18.0 & 12.4 & 9.9 & 100.0 & 54.9 & 18.7 & 70.3 \\
\hline $\mathrm{R} 20$ & 52221.8 & 53101.8 & 12.2 & 7.4 & 4.2 & 4.8 & 72.5 & 46.0 & 2.3 & 0.1 \\
\hline R30 & 67804.2 & 69230.2 & 15.6 & 15.2 & 6.0 & 9.8 & 62.8 & 52.2 & 2.0 & 0.3 \\
\hline $\mathrm{R} 40$ & 86734.0 & 90811.8 & 21.8 & 31.8 & 9.2 & 20.2 & 73.1 & 56.6 & 19.8 & 0.6 \\
\hline R50 & 91811.4 & 96350.4 & 28.6 & 33.8 & 15.4 & 21.4 & 67.0 & 58.1 & 9.8 & 1.1 \\
\hline & & & & & & $a x$ & $\begin{array}{r}100.0 \\
89.5\end{array}$ & 100.0 & $\begin{array}{r}92.1 \\
9 .\end{array}$ & 158.0 \\
\hline
\end{tabular}

Table 6: Results for $t$-CUARP, $\beta=0.5$

\begin{tabular}{|c|c|c|c|c|c|c|c|c|c|c|c|c|}
\hline & 2-CUARP & CUARP & $\# S 1$ & $\# S 2$ & $\# S 12$ & $\# S 21$ & \%serv. & \%exc. & increase 1 & increase 2 & increase & time \\
\hline AA & 14442.0 & 18722.0 & 42.0 & 39.0 & 23.0 & 28.0 & 97.6 & 63.0 & 22.9 & 30.3 & 10.5 & 58.9 \\
\hline $\mathrm{AB}$ & 1411.0 & 11946.0 & 45.0 & 26.0 & 19.0 & 24.0 & 97.3 & 60.6 & 88.2 & 107.0 & 39.4 & 47.9 \\
\hline $\mathrm{P}$ & 379.2 & 392.8 & 12.3 & 14.0 & 7.6 & 8.0 & 90.5 & 53.2 & 4.0 & 4.1 & 3.9 & 0.5 \\
\hline D16 & 1211.8 & 1220.4 & 5.0 & 7.9 & 3.2 & 3.8 & 79.5 & 53.1 & 0.9 & 0.7 & 0.7 & 0.2 \\
\hline D36 & 2443.9 & 2460.6 & 15.2 & 17.7 & 9.4 & 10.4 & 90.5 & 59.6 & 0.9 & 0.7 & 0.7 & 0.6 \\
\hline D64 & 3582.6 & 3616.6 & 31.0 & 29.9 & 16.6 & 20.6 & 94.5 & 61.0 & 1.0 & 1.2 & 1.1 & 9.3 \\
\hline D100 & 4810.6 & 4873.2 & 48.3 & 48.7 & 29.1 & 37.0 & 97.9 & 68.2 & 2.8 & 0.8 & 1.5 & 21.3 \\
\hline G16 & 10.3 & 12.2 & 1.4 & 2.4 & 0.6 & 0.9 & 94.4 & 32.0 & 18.9 & 5.7 & 14.3 & 0.1 \\
\hline G36 & 42.8 & 48.9 & 3.2 & 9.4 & 1.0 & 3.1 & 98.4 & 28.6 & 9.9 & 17.4 & 10.8 & 1.6 \\
\hline G64 & 87.6 & 99.7 & 12.2 & 12.1 & 6.4 & 4.3 & 100.0 & 46.6 & 12.8 & 20.6 & 12.8 & 11.2 \\
\hline G100 & 139.9 & 169.0 & 21.7 & 18.2 & 12.6 & 10.2 & 100.0 & 56.2 & 16.8 & 16.7 & 17.2 & 76.5 \\
\hline $\mathrm{R} 20$ & 52221.8 & 53061.8 & 11.6 & 8.0 & 4.6 & 5.8 & 72.5 & 50.7 & 2.1 & 3.4 & 2.1 & 0.2 \\
\hline R30 & 67804.2 & 69230.2 & 18.0 & 12.8 & 6.8 & 8.2 & 62.8 & 46.1 & 1.2 & 2.4 & 2.0 & 0.4 \\
\hline $\mathrm{R} 40$ & 86734.0 & 90539.0 & 24.0 & 29.6 & 14.6 & 23.4 & 73.1 & 71.8 & 4.3 & 7.7 & 19.5 & 0.9 \\
\hline R50 & 91811.4 & 96350.4 & 31.0 & 31.4 & 18.2 & 21.8 & 67.0 & 62.8 & 7.0 & 10.9 & 9.8 & 1.9 \\
\hline & & & & & & $\max$ & 100.0 & 100.0 & 88.2 & 107.0 & 85.7 & 158.0 \\
\hline & & & & & & average & 89.3 & 52.6 & 7.3 & 7.9 & 7.1 & 10.4 \\
\hline
\end{tabular}


Table 7: Results for $t$-CUARP, $\beta=1$

\begin{tabular}{|c|c|c|c|c|c|c|c|c|c|c|c|c|}
\hline & $n$-CUARP & CUARP & $\# S 1$ & $\# S 2$ & $\# S 12$ & $\# S 21$ & \%serv. & \%exc. & increase 1 & increase 2 & increase & time \\
\hline AA & 14442.0 & 24509.0 & 41.0 & 41.0 & 28.0 & 34.0 & 98.8 & 75.6 & 41.1 & 37.8 & 41.1 & 18.0 \\
\hline AB & 1411.0 & 17911.0 & 26.0 & 47.0 & 16.0 & 40.0 & 100.0 & 76.7 & 92.1 & 106.7 & 77.3 & 13.3 \\
\hline $\mathrm{P}$ & 379.2 & 392.5 & 12.2 & 14.1 & 6.9 & 7.5 & 91.1 & 44.0 & 5.3 & 2.3 & 3.5 & 0.7 \\
\hline D16 & 1211.8 & 1219.6 & 5.7 & 7.2 & 3.1 & 3.0 & 79.5 & 46.1 & 0.6 & 1.0 & 0.6 & 0.3 \\
\hline D36 & 2443.9 & 2460.3 & 14.0 & 19.0 & 8.3 & 10.6 & 90.8 & 56.7 & 0.6 & 0.9 & 0.7 & 0.9 \\
\hline D64 & 3582.6 & 3616.6 & 30.7 & 30.1 & 15.9 & 20.1 & 94.3 & 59.3 & 1.1 & 0.9 & 1.1 & 11.0 \\
\hline D100 & 4810.6 & 4873.2 & 52.4 & 44.4 & 29.0 & 32.8 & 97.7 & 63.8 & 1.6 & 1.8 & 1.5 & 23.3 \\
\hline G16 & 10.3 & 11.8 & 1.8 & 2.1 & 0.7 & 0.7 & 94.4 & 30.2 & 24.2 & 7.9 & 12.0 & 0.2 \\
\hline G36 & 42.8 & 44.9 & 5.4 & 7.2 & 1.7 & 1.6 & 98.4 & 21.4 & 11.1 & 1.2 & 4.3 & 2.3 \\
\hline G64 & 87.6 & 96.3 & 10.6 & 13.8 & 4.7 & 4.2 & 100.0 & 36.5 & 9.1 & 9.6 & 9.1 & 15.4 \\
\hline G100 & 139.9 & 155.0 & 19.7 & 20.2 & 9.9 & 9.6 & 100.0 & 48.0 & 10.9 & 9.4 & 11.0 & 100.2 \\
\hline $\mathrm{R} 20$ & 52221.8 & 53061.8 & 12.2 & 7.4 & 5.0 & 5.6 & 72.5 & 52.9 & 1.8 & 4.4 & 2.2 & 0.2 \\
\hline R30 & 67804.2 & 69190.2 & 15.4 & 15.4 & 6.8 & 10.8 & 62.8 & 56.1 & 2.5 & 1.8 & 1.9 & 0.8 \\
\hline $\mathrm{R} 40$ & 86734.0 & 90539.0 & 29.0 & 24.6 & 15.2 & 19.0 & 73.1 & 62.8 & 21.0 & 2.7 & 19.5 & 1.1 \\
\hline R50 & 91811.4 & $\begin{array}{l}96350.4 \\
\end{array}$ & 32.2 & 30.2 & 19.0 & 21.4 & 67.0 & 64.3 & 11.2 & 8.9 & 9.8 & 2.1 \\
\hline & & & & & & $\max$ & 100.0 & 80.0 & 100.0 & 106.7 & 85.7 & 291.9 \\
\hline & & & & & & average & 89.5 & 47.9 & 8.3 & 4.9 & 6.2 & 12.3 \\
\hline
\end{tabular}

Table 8: Instance summary after moving depots

\begin{tabular}{|c|c|c|c|c|c|c|}
\hline & \#inst. & \#vertices & $\left|R^{1}\right|$ & $\left|R^{2}\right|$ & $\left|S^{1}\right|$ & $\left|S^{2}\right|$ \\
\hline $\mathrm{AA}$ & 1 & 102 & 31 & 49 & 31 & 49 \\
\hline $\mathrm{AB}$ & 1 & 90 & 28 & 45 & 28 & 45 \\
\hline $\mathrm{P}$ & 24 & $7-50$ & $1-57$ & $2-49$ & $2-57$ & $2-49$ \\
\hline D16 & 9 & 16 & $5-9$ & $6-11$ & $6-9$ & $7-11$ \\
\hline D36 & 9 & 36 & $16-22$ & $12-22$ & $16-22$ & $12-22$ \\
\hline D64 & 9 & 64 & $26-42$ & $22-39$ & $26-42$ & $22-40$ \\
\hline D100 & 9 & 100 & $42-50$ & $50-55$ & $42-40$ & $51-56$ \\
\hline G16 & 9 & 16 & $\overline{0-2}$ & $0-4$ & $1-3$ & $0-4$ \\
\hline G36 & 9 & 36 & $2-7$ & $3-12$ & $2-8$ & $3-12$ \\
\hline G64 & 9 & 64 & $4-20$ & $6-17$ & $5-21$ & $6-17$ \\
\hline G100 & 9 & 100 & $8-27$ & \begin{tabular}{|c|}
$9-31$ \\
\end{tabular} & $9-27$ & $10-31$ \\
\hline $\mathrm{R} 20$ & 5 & 20 & $8-20$ & $8-20$ & $8-20$ & $9-20$ \\
\hline R30 & 5 & 30 & $18-30$ & $14-30$ & $19-30$ & $15-31$ \\
\hline R40 & 5 & 40 & $18-67$ & $22-48$ & $19-68$ & $23-48$ \\
\hline R50 & 5 & 50 & $31-48$ & $35-66$ & $31-49$ & $35-66$ \\
\hline
\end{tabular}

Table 9: Results for CUARP after moving depots

\begin{tabular}{|c|c|c|c|c|c|c|c|c|c|c|}
\hline & $n$-CUARP & CUARP & $\# S 1$ & $\# S 2$ & $\# S 12$ & $\# S 21$ & \%serv. & \%exc. & increase & time \\
\hline AA & 23570.0 & 23596.0 & 36.0 & 43.0 & 24.0 & 18.0 & 98.8 & 53.2 & 0.1 & 13.9 \\
\hline $\mathrm{AB}$ & 17657.0 & 17693.0 & 39.0 & 34.0 & 26.0 & 15.0 & 100.0 & 56.2 & 0.2 & 10.0 \\
\hline $\mathrm{P}$ & 380.3 & 390.9 & 11.2 & 14.5 & 6.6 & 7.3 & 91.8 & 54.7 & 4.3 & 0.3 \\
\hline D16 & 1221.2 & 1225.6 & 5.9 & 6.0 & 4.3 & 2.6 & 73.7 & 57.2 & 0.0 & 0.1 \\
\hline D36 & 1929.8 & 2072.8 & 19.7 & 12.2 & 10.6 & 7.0 & 87.9 & 54.5 & 0.1 & 0.6 \\
\hline D64 & 3622.3 & 3654.8 & 28.7 & 31.7 & 15.2 & 18.4 & 94.0 & 55.9 & 0.0 & 2.8 \\
\hline D100 & 5058.0 & 5106.2 & 39.6 & 55.4 & 24.8 & 30.2 & 94.9 & 58.0 & 0.0 & 14.5 \\
\hline G16 & 9.8 & 13.1 & 1.0 & 2.9 & 0.4 & 1.3 & 92.2 & 39.0 & 0.2 & 0.1 \\
\hline G36 & 38.7 & 45.9 & 4.7 & 7.8 & 3.0 & 3.9 & 100.0 & 54.9 & 0.2 & 1.8 \\
\hline G64 & 86.6 & 102.2 & 13.4 & 10.8 & 6.7 & 5.4 & 100.0 & 50.5 & 0.2 & 9.6 \\
\hline G100 & 147.0 & 167.6 & 20.1 & 20.2 & 10.8 & 10.4 & 100.0 & 52.3 & 0.2 & 103.8 \\
\hline R20 & 52781.8 & 53861.2 & 10.6 & 7.4 & 5.2 & 4.4 & 66.6 & 53.7 & 0.0 & 0.1 \\
\hline R30 & 63098.6 & 64668.2 & 13.8 & 20.2 & 8.4 & 11.4 & 68.5 & 56.2 & 0.0 & 0.2 \\
\hline R40 & 81383.0 & 83126.0 & 21.0 & 29.8 & 11.0 & 17.2 & 67.4 & 54.6 & 0.0 & 0.8 \\
\hline R50 & 82934.0 & 88777.4 & 30.8 & 36.8 & 16.6 & 15.6 & 71.4 & 48.2 & 0.2 & 1.5 \\
\hline & & & & & & & 100.0 & 88.9 & 0.9 & 185.1 \\
\hline & & & & & & average & 88.6 & 53.3 & 0.1 & 10.5 \\
\hline
\end{tabular}

Table 10: Instance summary with 3 depots

\begin{tabular}{|c|c|c|c|c|c|c|c|c|}
\hline & \#inst. & \#vertices & $\left|R^{1}\right|$ & $\left|R^{2}\right|$ & $\left|R^{3}\right|$ & $\left|S^{1}\right|$ & $\left|S^{2}\right|$ & $\left|S^{3}\right|$ \\
\hline $\mathrm{AA}$ & 1 & 102 & 40 & 22 & 17 & 41 & 23 & 17 \\
\hline AB & 1 & 90 & 46 & 11 & 17 & 46 & 12 & 17 \\
\hline $\mathrm{P}$ & 24 & $7-50$ & $1-43$ & $1-42$ & $0-22$ & $2-44$ & $1-42$ & $1-23$ \\
\hline D16 & 9 & 16 & $2-5$ & $6-10$ & $1-5$ & $2-6$ & $6-11$ & $2-6$ \\
\hline D36 & 9 & 36 & $10-18$ & $10-16$ & $7-11$ & $11-18$ & $10-16$ & $7-12$ \\
\hline D64 & 9 & 64 & $27-40$ & $8-22$ & $12-18$ & $27-41$ & $9-23$ & $13-18$ \\
\hline D100 & 9 & 100 & $36-61$ & $13-40$ & $19-29$ & $36-62$ & $13-40$ & $20-29$ \\
\hline G16 & 9 & 16 & $0-3$ & $0-2$ & $0-2$ & $1-3$ & $1-2$ & $0-2$ \\
\hline G36 & 9 & 36 & $1-8$ & $2-10$ & $1-6$ & $1-8$ & $2-10$ & $1-6$ \\
\hline G64 & 9 & 64 & $2-13$ & $4-14$ & $2-11$ & $2-13$ & $5-15$ & $2-11$ \\
\hline G100 & 9 & 100 & $6-23$ & $7-24$ & $5-14$ & $6-24$ & $8-24$ & $5-14$ \\
\hline $\mathrm{R} 20$ & 5 & 20 & $6-23$ & $3-24$ & $3-14$ & $6-24$ & $4-24$ & $4-14$ \\
\hline R30 & 5 & 30 & $6-40$ & $2-24$ & $3-15$ & $6-40$ & $3-24$ & 415 \\
\hline $\mathrm{R} 40$ & 5 & 40 & $6-48$ & $2-31$ & $3-27$ & $6-48$ & $3-31$ & $4-415$ \\
\hline R50 & 5 & 50 & $6-53$ & $2-44$ & $3-28$ & $6-54$ & $3-45$ & $4-415$ \\
\hline
\end{tabular}


Table 11: Results for CUARP with 3 depots

\begin{tabular}{|c|c|c|c|c|c|c|c|c|}
\hline & $n$-CUARP & CUARP & $\# S$ & $\# \operatorname{Sexc}$ & \%serv. & \%exc. & increase & time \\
\hline AA & 25481.0 & 25513.0 & 42.0 & 16.0 & 51.9 & 38.1 & 0.1 & 41.8 \\
\hline $\mathrm{AB}$ & 20898.0 & 20930.0 & 44.0 & 19.0 & 58.7 & 43.2 & 0.2 & 36.7 \\
\hline $\mathrm{P}$ & 433.8 & 445.6 & 14.3 & 5.4 & 46.3 & 39.2 & 3.9 & 1.0 \\
\hline D16 & 1379.2 & 1388.1 & 7.7 & 3.1 & 46.5 & 41.2 & 0.7 & 0.1 \\
\hline D36 & 2661.4 & 2681.4 & 17.3 & 7.2 & 47.4 & 41.8 & 1.0 & 1.0 \\
\hline D64 & 4068.2 & 4015.3 & 36.8 & 13.6 & 57.2 & 36.9 & 0.6 & 9.3 \\
\hline D100 & 5387.2 & 5438.0 & 54.6 & 18.3 & 54.7 & 33.7 & 1.1 & 55.5 \\
\hline G16 & 15.6 & 18.0 & 2.9 & 1.3 & 66.7 & 42.8 & 16.8 & 0.1 \\
\hline G36 & 52.4 & 61.3 & 6.6 & 1.9 & 54.5 & 26.4 & 15.5 & 3.7 \\
\hline G64 & 111.8 & 121.7 & 12.2 & 4.4 & 49.4 & 35.2 & 6.9 & 24.6 \\
\hline G100 & 171.6 & 191.7 & 21.4 & 6.7 & 50.1 & 28.7 & 11.3 & 248.1 \\
\hline $\mathrm{R} 20$ & 60528.6 & 60537.8 & 15.4 & 5.6 & 58.5 & 33.0 & 0.0 & 0.2 \\
\hline R30 & 88964.0 & 88986.0 & 16.4 & 5.2 & 35.0 & 30.8 & 0.0 & 0.5 \\
\hline $\mathrm{R} 40$ & 115561.6 & 115582.8 & 34.6 & 13.8 & 45.9 & 35.9 & 0.0 & 1.4 \\
\hline R50 & 128557.6 & 128587.0 & 40.4 & 18.4 & 43.0 & 46.5 & 0.0 & 3.2 \\
\hline & & & & $\max$ & 100.0 & 100.0 & 80.0 & 546.3 \\
\hline & & & & average & 50.6 & 36.7 & 4.9 & 27.2 \\
\hline
\end{tabular}

Table 12: CUARP G16 results with increasing size of shared sets

\begin{tabular}{|c|rrrrr|}
\hline \multicolumn{1}{l}{} & $n$-CUARP & CUARP-25 & CUARP & CUARP-75 & CUARP-100 \\
\hline G1 & 1 & 1 & 1 & 1 & 2 \\
\hline G2 & 3 & 6 & 6 & 6 & 15 \\
\hline G3 & 4 & 6 & 6 & 6 & 8 \\
\hline G4 & 14 & 17 & 17 & 17 & 27 \\
\hline G5 & 10 & 13 & 13 & 13 & 25 \\
\hline G6 & 11 & 14 & 14 & 14 & 30 \\
\hline G7 & 13 & 28 & 28 & 34 & 47 \\
\hline G8 & 14 & 15 & 17 & 17 & 28 \\
\hline
\end{tabular}




\section{Conclusions}

In this paper, we have introduced two variants of the Collaboration Uncapacitated Arc Routing Problem. This is a profitable arc routing problem with multiple depots, where carriers may collaborate to improve the profit gained. An integer linear programming formulation with binary and integer variables has been presented, as well as a branchand-cut algorithm. In the CUARP the goal is the maximization of the total profit of the coalition. The profit gained by the coalition of carriers never decreases with respect to the case without collaboration. Individual carriers, however, may lose profit in the collaboration scheme modeled by the CUARP. For this reason, we considered a variant where carriers may set thresholds on the profit gained in the collaborative scheme. This variant allocates the profit gained by the coalition in a more balanced way. Interestingly, we analyzed the cooperative game associated with the CUARP and noted that in the case of two carriers the $t$-CUARP profit allocation is stable because it belongs to the core of the game. Moreover, collaboration may produce an arbitrary large increase of the profit. A set of benchmark instances were generated and the results of extensive computational experiments presented and analyzed.

For future research, attention should be focused on the extension of the models proposed to include real-life features of the problem such as time and capacity constraints. Another direction concerns the design of heuristic algorithms for the solution of large instances.

\section{Acknowledgements}

The research of the first author has been partially supported by the Spanish Ministry of Economy and Competitivity through grant MTM2012-36163-C06-05 and by ERDF funds. This support is gratefully acknowledged. The authors would like to express their appreciation for the valuable and insightful comments made by an anonymous reviewer.

\section{References}

[1] R. Agarwal and Ö. Ergun. Network design and allocation mechanisms for carrier alliances in liner shipping. Operations Research, 58(6):1726-1742, 2010.

[2] R. Agarwal, Ö. Ergun, L. Houghtalen, and O. Ö. Özener. Collaboration in cargo transportation. In Optimization and Logistics Challenges in the Enterprise, pages 373-409. Springer, 2009.

[3] D. Ahr. Contributions to Multiple Postmen Problems. PhD dissertation, Departament of Computer Science, Heidelberg University, Heidelberg, Germany. 2004. 
[4] J. Aráoz, E. Fernández, and C. Franquesa. The clustered prize-collecting arc routing problem. Transportation Science, 43(3):287-300, 2009.

[5] J. Aráoz, E. Fernández, and O. Meza. Solving the prize-collecting rural postman problem. European Journal of Operational Research, 196(3):886-896, 2009.

[6] J. Aráoz, E. Fernández, and C. Zoltan. Privatized rural postman problems. Computers $\&$ Operations Research, 33(12):3432-3449, 2006.

[7] C. Archetti, G. Guastaroba, and M. Speranza. An ilp-refined tabu search for the directed profitable rural postman problem. Discrete Applied Mathematics, 163:3-16, 2014.

[8] C. Archetti and M. G. Speranza. Arc routing problems with profits. in Arc Routing: Problems, Methods, and Applications. Á. Corberán and G. Laporte (eds.) MOS-SIAM series on Optimization, 281-297, 2015.

[9] J.-F. Audy, S. D'Amours, and L.-M. Rousseau. Cost allocation in the establishment of a collaborative transportation agreement: an application in the furniture industry. Journal of the Operational Research Society, 62(6):960-970, 2011.

[10] J. M. Belenguer and E. Benavent. The capacitated arc routing problem: Valid inequalities and facets. Computational Optimization and Applications, 10(2):165187, 1998.

[11] E. Benavent, A. Corberán and J.M. Sanchis. Linear programming based methods for solving arc routing problems. In Arc Routing: Theory, Solutions and Applications. M. Dror (ed.) Kluwer Academic Publishers, 231-275, 2000.

[12] N. Christofides, V. Campos, A. Corberán, and E. Mota. An algorithm for the rural postman problem on a directed graph. Mathematical Programming Study, 26: 155-166. Springer, 1986.

[13] Á. Corberán, E. Fernández, C. Franquesa, and J. M. Sanchis. The windy clustered prize-collecting arc-routing problem. Transportation Science, 45(3):317-334, 2011.

[14] A. Corberán and J. Sanchis. A polyhedral approach to the rural postman problem. European Journal of Operational Research, 79(1):95-114, 1994.

[15] T. S. Driessen. A survey of consistency properties in cooperative game theory. SIAM review, 33(1):43-59, 1991.

[16] H. A. Eiselt, M. Gendreau, and G. Laporte. Arc routing problems, part i: The chinese postman problem. Operations Research, 43(2):231-242, 1995.

[17] H. A. Eiselt, M. Gendreau, and G. Laporte. Arc routing problems, part ii: The rural postman problem. Operations Research, 43(3):399-414, 1995.

[18] Ö. Ergun, G. Kuyzu, and M. Savelsbergh. Reducing truckload transportation costs through collaboration. Transportation Science, 41(2):206-221, 2007. 
[19] Ö. Ergun, G. Kuyzu, and M. Savelsbergh. Shipper collaboration. Computers \& Operations Research, 34(6):1551-1560, 2007.

[20] M. A. Figliozzi. Analysis and evaluation of incentive-compatible dynamic mechanisms for carrier collaboration. Transportation Research Record: Journal of the Transportation Research Board, 1966(1):34-40, 2006.

[21] B. S. Fugate, B. Davis-Sramek, and T. J. Goldsby. Operational collaboration between shippers and carriers in the transportation industry. The International Journal of Logistics Management, 20(3):425-447, 2009.

[22] R. E. Gomory and T. C. Hu. Multi-terminal network flows. Journal of the Society for Industrial \& Applied Mathematics, 9(4):551-570, 1961.

[23] M. Göthe-Lundgren, K. Jörnsten, and P. Värbrand. On the nucleolus of the basic vehicle routing game. Mathematical Programming, 72(1):83-100, 1996.

[24] D. Gusfield. Very simple methods for all pairs network flow analysis. SIAM Journal Applied Mathematics 19, 1, 143-555, 1990.

[25] A. Hertz, G. Laporte, and P. N. Hugo. Improvement procedures for the undirected rural postman problem. INFORMS Journal on Computing, 11(1):53-62, 1999.

[26] M. A. Krajewska, H. Kopfer, G. Laporte, S. Ropke, and G. Zaccour. Horizontal cooperation among freight carriers: request allocation and profit sharing. Journal of the Operational Research Society, 59(11):1483-1491, 2008.

[27] J. K. Lenstra and A. H. G. Rinnooy Kan. On general routing problems. Networks, 6(3):273-280, 1976.

[28] R. Mason, C. Lalwani, and R. Boughton. Combining vertical and horizontal collaboration for transport optimisation. Supply Chain Management: An International Journal, 12(3):187-199, 2007.

[29] M. D. Meyer, S. Campbell, D. Leach, and M. Coogan. Collaboration: The key to success in transportation. Transportation Research Record: Journal of the Transportation Research Board, 1924(1):153-162, 2005.

[30] O. Ö. Özener, Ö. Ergun, and M. Savelsbergh. Lane-exchange mechanisms for truckload carrier collaboration. Transportation Science, 45(1):1-17, 2011.

[31] H. J. Quak. Improving urban freight transport sustainability by carriers-best practices from the Netherlands and the EU project citylog. Procedia-Social and Behavioral Sciences, 39:158-171, 2012. 